\title{
INTERFACE LOSSES IN MULTIMATERIAL RESONATORS
}

\author{
L.G. Villanueva ${ }^{1,2, *}$, B. Amato ${ }^{l}$, T. Larsen $^{l}$ and S. Schmid ${ }^{l}$ \\ ${ }^{1}$ DTU Nanotech, Technical University of Denmark (DTU), Lyngby, Denmark \\ ${ }^{2}$ Advanced NEMS Group, École Polytechnique Fédérale de Lausanne (EPFL), Lausanne, Switzerland
}

\begin{abstract}
We present an extensive study shedding light on the role of surface and bulk losses in micromechanical resonators. We fabricate thin silicon nitride membranes of different sizes and we coat them with different thicknesses of metal. We later characterize the 81 lowest out-of-plane flexural vibrational modes to achieve a total of more than 3000 experimental points that allow us to quantify the contribution of surface and volume intrinsic (material related) losses in MEMS resonators. We conclude that the losses in the interface between silicon nitride and aluminum is a very important contributor to the overall energy loss.
\end{abstract}

\section{INTRODUCTION}

With very high quality factors (Qs) values (up to $10^{7}$ ) at room temperature and $Q \cdot f$ products (above $10^{13} \mathrm{~Hz}$ ), stoichiometric $\mathrm{Si}_{3} \mathrm{~N}_{4}$ membranes [1] and strings [2] have become a centerpiece of many research projects, particularly in opto-mechanics [3, 4]. Recently it has been shown that metallized membranes enable the design of exciting new opto-electro-mechanical systems that allow e.g. the optical detection of electrical signals with unprecedented sensitivity $[5,6]$. For these applications and for MEMS resonators in general there has been a continuous effort to find materials and systems that provide as high Qs as possible. The thorough understanding of the underlying loss mechanisms is crucial to optimize $\mathrm{Q}$.

Q can be defined as the ratio between the energy stored in a resonator over the energy loss every cycle. Due to their large intrinsic residual stress, resonating membranes and string are able to store more energy, thus increasing $\mathrm{Q}$ even though dissipated energy per cycle remains the same. Models based on this idea, considering only material losses, are able to reproduce the behavior of $\mathrm{Q}$ as a function of mode number (whenever neither of the indexes is lower than 3), and even suggest ways to control extra losses for multi-material resonators [7]. However, the data reported in the literature does not provide information on the relative importance of surface vs. bulk losses for these systems. In this work, we quantify both bulk and surface losses, evidencing the importance of proper surfaces, not only in the physical boundaries of the resonator, but also in the interface between different materials.

\section{FABRICATION}

We fabricate our membranes following the simple procedure outlined in Figure 1. We start by defining a set of $\mathrm{Si}_{3} \mathrm{~N}_{4}$ square membranes $(L=250,500$, and $1000 \mu \mathrm{m}$; $t_{S i_{3} N_{4}}=50,100$, and $200 \mathrm{~nm}$ ). Our initial substrates are double side polished P-doped silicon wafers $(100 \mathrm{~mm}$ in diameter) where the stoichiometric silicon nitride is deposited via Low Pressure Chemical Vapor Deposition (LPCVD) at $850^{\circ} \mathrm{C}$. The backside is then patterned to define windows for membrane release, by standard photolithography (using flat alignment for a more precise definition of the membrane sizes) and dry etching, followed by $\mathrm{KOH}$ micromachining of Si wafers ( $\mathrm{KOH}$ solution $40 \%$ in weight at $85^{\circ} \mathrm{C}$ ), followed by a cleaning in a neutralization bath to remove $\mathrm{KOH}$ crystals residues. Aluminum is then deposited on top of some of the samples $\left(t_{S i_{3} N_{4}}=\right.$ 50,100 , and $200 \mathrm{~nm}$ ) by e-beam evaporation at $10^{-6} \mathrm{mbar}$ at rates ranging between 0.5 and $1 \mathrm{~nm} / \mathrm{s}$. In the last step all samples are annealed at $400^{\circ} \mathrm{C}$ in order to help $\mathrm{Al}$ reflow, reduce intrinsic losses and make stresses uniform [7].
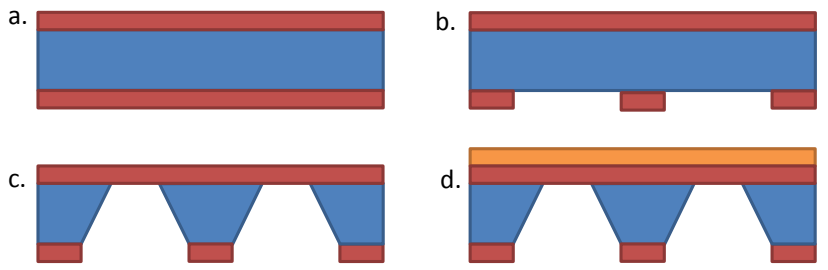

Silicon

LPCVD Silicon Nitride

Aluminum

Figure 1: Fabrication process flow for the membrane resonators. A layer of $L P C V D$ stoichiometric $\mathrm{Si}_{3} \mathrm{~N}_{4}$ is deposited on a Si wafer (a). This layer is patterned (b) on the backside to define the windows for the subsequent $\mathrm{Si}$ anisotropic etching using $\mathrm{KOH}$ (c). The front side is metallized with the desired thickness of aluminum using $e$ beam evaporation $(d)$.

\section{CHARACTERIZATION}

Characterization is performed in vacuum $(P \leq$ $10^{-5} \mathrm{mbar}$ ), at room temperature. The silicon chips with membranes are located on top of a piezo-shaker disc for actuation purposes. To detect the motion of the membranes, a Polytec Laser-Doppler vibrometer with a bandwidth of 24 $\mathrm{MHz}$ is used. We study the 81 lower out-of-plane flexural vibrational modes measuring their resonance frequencies and quality factors. To determine the resonance frequencies, standard frequency sweeps are performed using a Zurich Instruments HF2LI lock-in amplifier. To determine the quality factor, we compare two different methods on the first membrane. We extract the quality factor from the frequency sweep scan performed with the lock-in amplifier and from ring-down experiments. We do this 5 times per mode for up to 20 modes and we observe that the variance of the 
experiment is larger than the difference between both methods. Therefore, for the rest of the modes we extract the quality factor from the same frequency sweep data we use to determine the frequency. This reduces considerably the experiment time.

Taking into account the different membrane geometries (both lateral and thickness) we obtain in total more than 3000 experimental points for both frequency and quality factor.

\section{Reactive material properties}

As expected for structures with such extreme aspect ratios, the frequency of the modes is very accurately described by standard thin plate theory (see Figure 2):

$$
f_{n, m}=\frac{1}{2 L} \sqrt{v_{e f f}\left(n^{2}+m^{2}\right)} ;
$$

where $v_{\text {eff }}$ is the effective speed of sound for each particular multimaterial stack and it is given by Eq. 2:

$$
v_{e f f}=\sqrt{\frac{\sigma_{S i_{3} N_{4}} t_{S i_{3} N_{4}}+\sigma_{A l} t_{A l}}{\rho_{S i_{3} N_{4}} t_{S i_{3} N_{4}}+\rho_{A l} t_{A l}}}
$$

Using equations (1) and (2), it is possible to use the measured frequency values to estimate the residual stress for both stoichiometric $\mathrm{Si}_{3} \mathrm{~N}_{4}$ and $\mathrm{Al}$, obtaining $\sigma_{\mathrm{Si}_{3} N_{4}} \approx$ $1.125 \mathrm{GPa}$ and $\sigma_{A l} \approx 100 \mathrm{MPa}$. The positive sign is kept to identify both intrinsic stresses being of tensile nature. In Figure 3 it is possible to see how these two values for stress allow us to recover all $12 v_{\text {eff }}$.

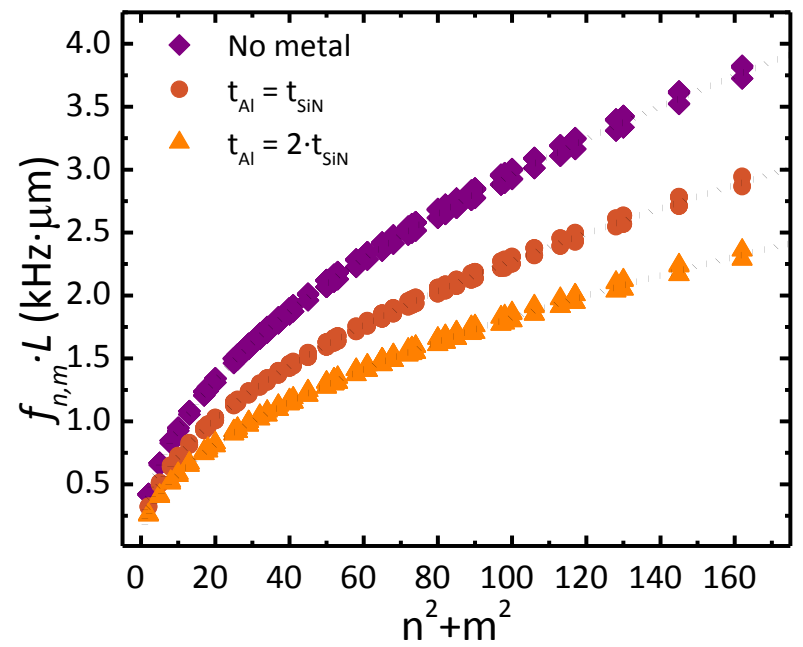

Figure 2: Experimentally obtained frequencies (scattered points, scaled by the length) for the 81 first flexural modes $v$ s. mode number for 13 membranes with different dimensions. Only three theoretical curves (dotted black lines) are expected, depending on the different effective speed of sound $\left(v_{\text {eff }}\right)$. A remarkable agreement to theory is obtained throughout the experimental data.

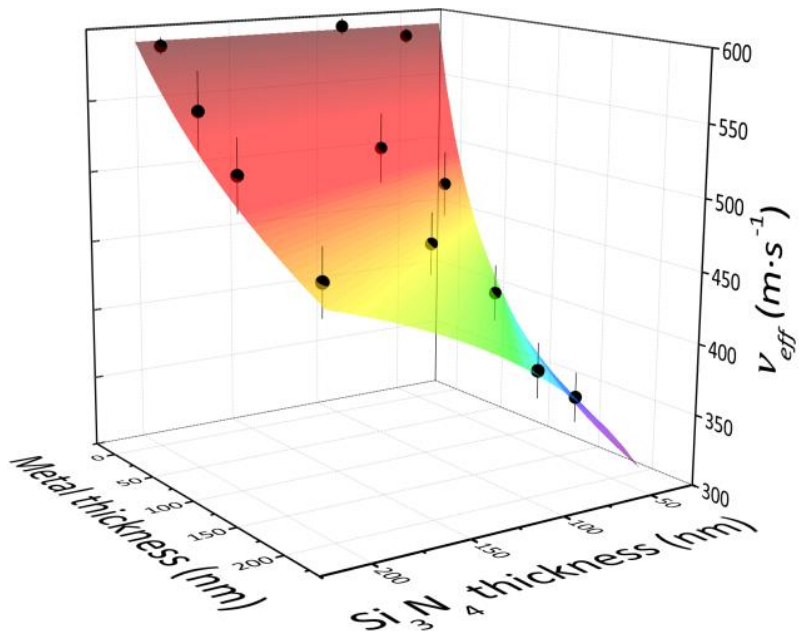

Figure 3: Using the 12 different material configurations for the fabricated membranes, it is possible to extract the residual stress and densities for both $\mathrm{Si}_{3} \mathrm{~N}_{4}$ and Al. We do this by fitting the experimentally measured values for $v_{\text {eff }}$.

\section{Dissipative material properties}

In order to analyze the energy losses in the characterized resonators, we first separate the different modes into those limited by anchor losses (radiation of energy to the clamping substrate) and those limited by internal (material) losses. This is done following the same semi-empirical rule suggested in [7], which states that all the modes with any of the indexes smaller than $3(i \vee j<3)$. Figure 5 shows how the overall dispersion in the quality factors distribution is reduced considerably if we only consider modes limited by material losses (solid scattered data).

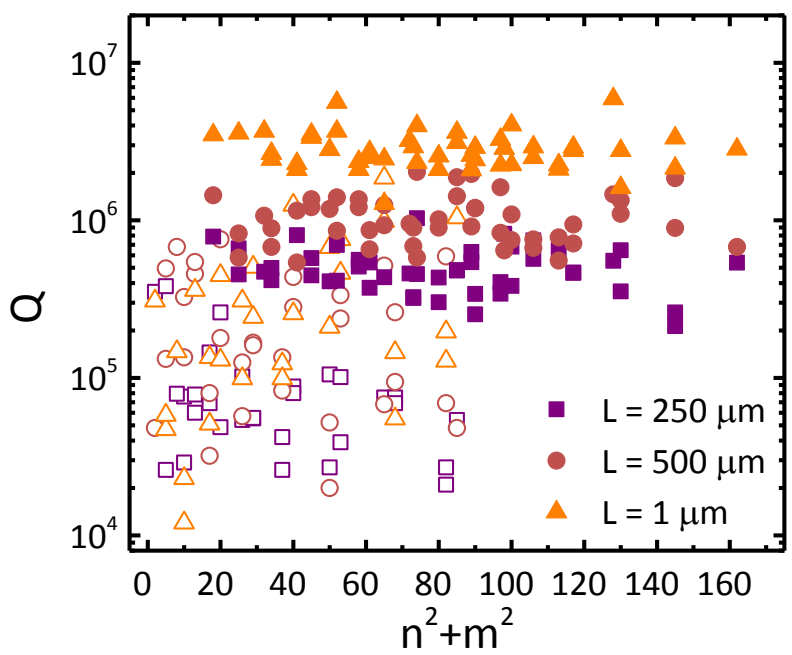

Figure 4: Experimentally obtained quality factors for the 81 first flexural modes vs. mode number for 3 membranes with the same $t_{S_{3} N_{4}}=50 \mathrm{~nm}$ and different lengths. Following [7] we separate between the modes which are being limited by anchoring losses (outlined scattered symbols) and those that are limited by intrinsic material losses (solid scattered symbols). 
Next, we use a model similar to those already established in the literature $[2,7]$ to account for material-related energy losses (in the subset of modes described above). This consists on the use of a Zener model, where the Young's modulus of the material has a real and an imaginary part, the former one being of reactive nature and keeping the phase of strain and stress fields the same; while the latter is dissipative because it creates a phase lag between the strain and stress fields.

We start by considering only bulk losses for both materials. This model is a modification of the one presented elsewhere [7], accounting for the fact that the metal thickness will cause the neutral axis to shift with respect to the monomaterial case. The results of our analysis using this model can be seen in Figure 5.

We find that the resonators purely made of $\mathrm{Si}_{3} \mathrm{~N}_{4}$ can be represented by an imaginary Young's modulus of $\approx 0.2 \mathrm{GPa}$, which means that the behavior in this type of membranes that are characterized during our experiments can be purely explained using bulk losses. In Figure 5, top-left, it is possible to see how all the experimental data group together around the same value for the imaginary modulus.

However, when we put metal layers of different thicknesses, it is clearly visible that we need a more complex model. Figure 5 shows that for any of the nitride thicknesses the experimental counts show a mean value that depends strongly on the thickness of metal that the membrane has. This would mean that our deposited metal presents different intrinsic (bulk) losses depending on its thickness, which is very unrealistic.

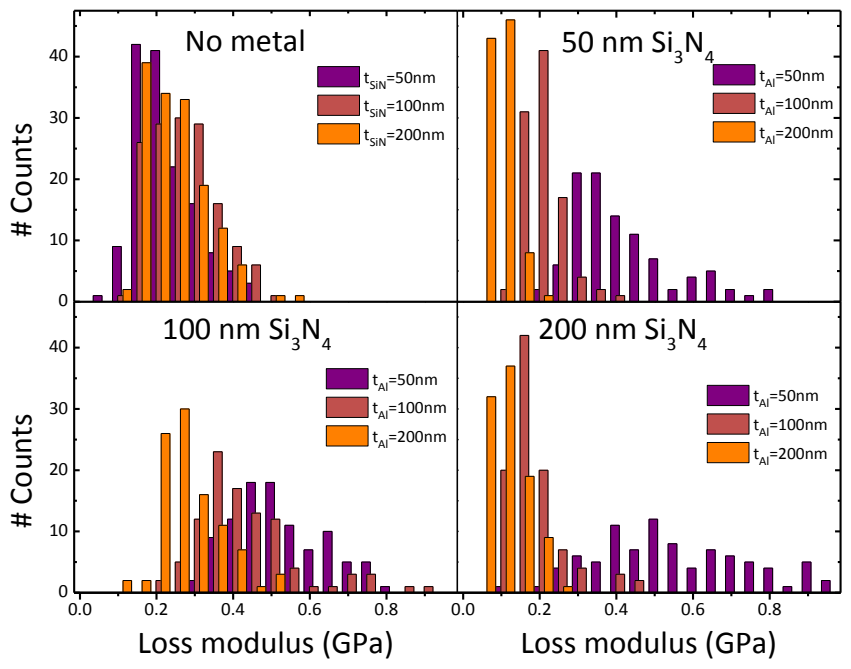

Figure 5: Histograms of the calculated imaginary components of the Young's modulus for $\mathrm{Si}_{3} \mathrm{~N}_{4}$ (top-left) and Aluminum (rest of graphs) for different thicknesses. It is clear that a model considering bulk losses describes well our $\mathrm{Si}_{3} \mathrm{~N}_{4}$ resonators (all counts are grouped together around the same value for the loss modulus), but not those that are multi-material (the event counts are dispersed, having mean values at different places depending on the metal thickness). This implies that an adjustment to the loss modulus needs to be done for different metal thicknesses.
Instead, our approach consists to include surface losses in addition to the already considered. In order to do this we follow the concept introduced in [8] and we consider the presence of extremely thin layer(s) at the interface between $\mathrm{Al}$ and $\mathrm{Si}_{3} \mathrm{~N}_{4}$ and at the top of the $\mathrm{Al}$ layer. This layers have the same reactive elastic properties as Aluminum but they have different dissipative elastic modulus. We later scale out the thickness of this superficial layers, and that is why the surface loss modulus have units of $\mathrm{N} / \mathrm{m}$. The bottom surface of $\mathrm{Si}_{3} \mathrm{~N}_{4}$ is not considered because, as it can be seen in the top-left of Figure 5, a model accounting only for bulk losses can explain the measured data. This point, however needs to be treated delicately as this conclusion contradicts previously reported data, where people showed that surface losses are also important for $\mathrm{Si}_{3} \mathrm{~N}_{4}$ 1-D structures [8-10]. Further measurements are being performed to check that the conclusion we reach is correct.

With the model we have described, we find that we are able to fit the loss parameters to:

$$
\begin{aligned}
& E_{\text {loss }, S_{3} N_{4}}=0.2 \pm 0.1 \mathrm{GPa}, \\
& E_{\text {loss }, A l}=0.1 \pm 0.05 \mathrm{GPa}, \\
& E_{\text {al-top }}^{*}=2 \pm 0.5 \frac{\mathrm{N}}{\mathrm{m}^{\prime}} \\
& E_{\text {interface }}^{*}=20 \pm 5 \frac{\mathrm{N}}{\mathrm{m}^{\prime}}
\end{aligned}
$$

with a confidence interval close to $75 \%$. Figure 6 shows the difference between theory and experiment for all modes of metallized membranes limited by intrinsic losses. The estimations for the model are made using the central value of the parameters in Equation (3).

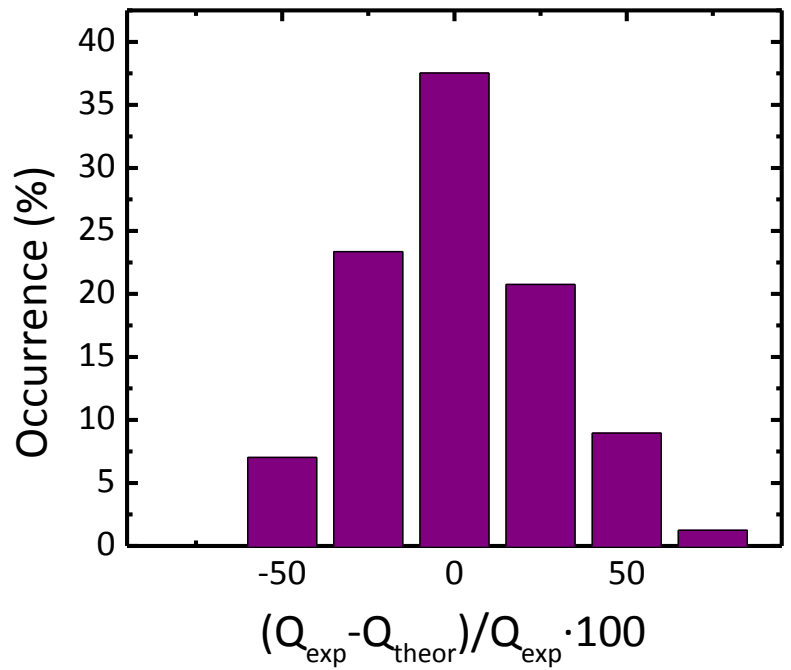

Figure 6: Relative difference between experimentally measured quality factors and theoretically estimated quality factors using our model and mean values for the fitted parameters. Close to 900 data points are considered in this plot, including values for the quality factors which differ in some orders of magnitude. 
Figure 7 shows graphically how our model predicts the quality factor for a set of four membranes of $1 \mathrm{~mm}$ lateral dimensions and with a thickness of $\mathrm{Si}_{3} \mathrm{~N}_{4}$ of $50 \mathrm{~nm}$.

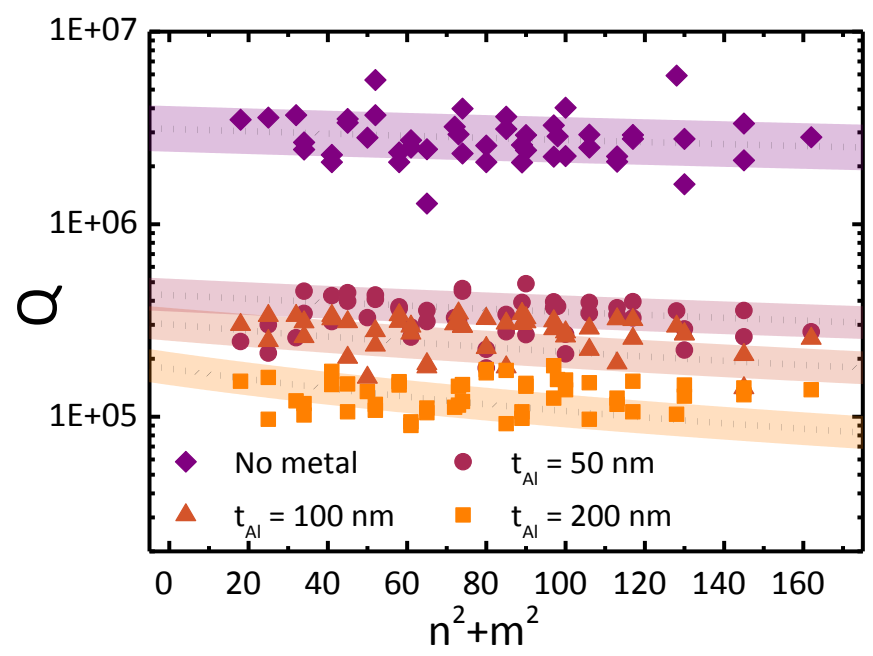

Figure 7: Experimentally obtained quality factors (scattered data) for $1 \mathrm{~mm}$ long membranes with $t_{S_{3} \mathrm{~N}_{4}}=$ $50 \mathrm{~nm}$ and different metal thicknesses. Dotted black lines show the theoretical prediction using a model that accounts for surface losses. Shaded regions correspond to the ( $75 \%)$ confidence intervals of the fit.

As a conclusion, we have quantified the importance of interface losses in multimaterial resonators which is of the utmost importance when piezoelectric [11, 12] and/or piezometallic [13] transduction are utilized or when a metal layer constitutes a functional part of the device, e.g. $\mathrm{H}_{2}$ absorption into Pd [14]. In addition, are opening an important and interesting line or research to optimize the interfaces (by for example pre-deposition surface treatments) and the metallic material [15] in order to minimize dissipation.

\section{ACKNOWLEDGEMENTS}

The authors would like to acknowledge Prof. A. Boisen for her support and discussions, and the staff in DTUDanchip for help in the fabrication of the membranes.

\section{REFERENCES}

[1] D.J. Wilson, C.A. Regal, S.B. Papp, et al., "Cavity Optomechanics with Stoichiometric SiN Films", Phys Rev Lett, vol. 103, pp. 207204, 2009.

[2] S. Schmid, K.D. Jensen, K.H. Nielsen, et al., "Damping mechanisms in high-Q micro and nanomechanical string resonators", Phys Rev B, vol. 84, pp. 165307, 2011.

[3] T.P. Purdy, R.W. Peterson, and C.A. Regal, "Observation of Radiation Pressure Shot Noise on a Macroscopic Object", Science, vol. 339, pp. 801-804, 2013.
[4] E. Gavartin, P. Verlot, and T.J. Kippenberg, "A hybrid on-chip optomechanical transducer for ultrasensitive force measurements", Nat Nano, vol. 7, pp. 509-514, 2012.

[5] T. Bagci, A. Simonsen, S. Schmid, et al., "Optical detection of radio waves through a nanomechanical transducer", Arxiv, vol. pp. 1307.3467, 2013.

[6] R.W. Andrews, R.W. Peterson, T.P. Purdy, et al., "Reversible and efficient conversion between microwave and optical light", Arxiv, vol. pp. 1310.5276, 2013.

[7] P.L. Yu, T.P. Purdy, and C.A. Regal, "Control of Material Damping in High-Q Membrane Microresonators", Phys Rev Lett, vol. 108, pp. 083603, 2012.

[8] K.Y. Yasumura, T.D. Stowe, E.M. Chow, et al., "Quality factors in micron- and submicron-thick cantilevers", $J$ Microelectromech S, vol. 9, pp. 117-125, 2000.

[9] S.S. Verbridge, J.M. Parpia, R.B. Reichenbach, et al., "High quality factor resonance at room temperature with nanostrings under high tensile stress", J Appl Phys, vol. 99, pp. 2006.

[10] S.S. Verbridge, D.F. Shapiro, H.G. Craighead, et al., "Macroscopic tuning of nanomechanics: Substrate bending for reversible control of frequency and quality factor of nanostring resonators", Nano Lett, vol. 7, pp. 1728-1735, 2007.

[11] L.G. Villanueva, R.B. Karabalin, M.H. Matheny, et al., "A Nanoscale Parametric Feedback Oscillator", Nano Lett, vol. 11, pp. 5054-5059, 2011.

[12] L.G. Villanueva, E. Kenig, R.B. Karabalin, et al., "Surpassing Fundamental Limits of Oscillators Using Nonlinear Resonators", Phys Rev Lett, vol. 110, pp. 177208, 2013.

[13] L.G. Villanueva, R.B. Karabalin, M.H. Matheny, et al., "Nonlinearity in nanomechanical cantilevers", Phys Rev $B$, vol. 87, pp. 024304, 2013.

[14] J. Henriksson, L.G. Villanueva, and J. Brugger, "Ultralow power hydrogen sensing based on a palladiumcoated nanomechanical beam resonator", Nanoscale, vol. 4, pp. 5059-5064, 2012.

[15] S. Schmid, T. Bagci, E. Zeuthen, et al., "Graphene on silicon nitride for optoelectromechanical micromembrane resonators", Arxiv, vol. pp. 1305.5890, 2013.

\section{CONTACT}

*L.G. Villanueva, Tel.: +41 21693 1187;

Guillermo.Villanueva@epfl.ch 